\title{
A disease causing ATLASTIN 3 mutation affects multiple endoplasmic reticulum-related pathways
}

\author{
Laura Behrendt $^{1} \cdot$ Ingo Kurth $^{2} \mathbb{D} \cdot$ Christoph Kaether $^{1} \mathbb{D}$
}

Received: 21 June 2018 / Revised: 9 January 2019 / Accepted: 10 January 2019 / Published online: 21 January 2019

(c) The Author(s) 2019

\begin{abstract}
Atlastins (ATLs) are membrane-bound GTPases involved in shaping of the endoplasmic reticulum (ER). Mutations in ATL1 and ATL3 cause spastic paraplegia and hereditary sensory neuropathy. We here show that the sensory neuropathy causing ATL3 Y192C mutation reduces the complexity of the tubular ER-network. ATL3 Y192C delays ER-export by reducing the number of ER exit sites, reduces autophagy, fragments the Golgi and causes malformation of the nucleus. In cultured primary neurons, ATL3 Y192C does not localize to the growing axon, resulting in axon growth deficits. Patient-derived fibroblasts possess a tubular ER with reduced complexity and have a reduced number of autophagosomes. The data suggest that the disease-causing ATL3 Y192C mutation affects multiple ER-related pathways, possibly as a consequence of the distorted ER morphology.
\end{abstract}

Keywords Atlastin $\cdot$ Hereditary spastic paraplegia $\cdot$ Endoplasmic reticulum $\cdot$ Secretory transport

\section{Introduction}

Length-dependent axonopathies comprise a large group of hereditary disorders affecting both afferent and efferent neurons with exceptional long axons [1,2]. Among this large group, peripheral motor neurons are predominantly affected in Charcot-Marie-Tooth (CMT) neuropathies and distal pure hereditary motor neuropathies (HMN), whereas sensory neurons are affected in hereditary sensory and autonomic neuropathies (HSAN). Upper motor neurons, in contrast, are affected in hereditary spastic paraplegias (HSP) [3]. Common to these heterogeneous groups of neurological disorders is the length-dependent axonopathy of projecting neurons.

Electronic supplementary material The online version of this article (https://doi.org/10.1007/s00018-019-03010-x) contains supplementary material, which is available to authorized users.

Christoph Kaether

ckaether@fli-leibniz.de

1 Leibniz Institut für Alternsforschung-Fritz Lipmann Institut, Beutenbergstr. 11, 07745 Jena, Germany

2 Institute of Human Genetics, Medical Faculty, RWTH Aachen University, 52074 Aachen, Germany
Causative mutations for axonopathies have been identified in genes functioning in several neuronal processes, among them intracellular transport, endosomal function and others (for review see Refs. [1, 3, 4]). Of interest are a number of axonopathy-causing genes involved in the architecture of the endoplasmic reticulum (ER). Many of them code for proteins with a reticulon-domain or reticulon-like domain, namely ARL6IP1, ATL1, ATL3, FAM134B, REEP1, RTN2 and SPAST (reviewed in Ref. [1]). The Atlastins (ATLs), gene products from ATL1-3, are large dynamin-related GTPases that are involved in ER network formation $[5,6]$ and maintenance [7]. ATL1 was identified as the gene product of the ATL1/SPG3A gene locus [8] and is mutated in app. 10\% of autosomal-dominant pure/uncomplicated forms of HSP [9] and in rare cases of HSAN1 [10]. Two heterozygous mutations causing HSAN were described in ATL3, Y192C [11] and P338R [12]. Both mutations are defective in dimerization and fusion, resulting in aberrant bundling of ER tubules [13].

The role of ATLs in ER-membrane fusion is well established. ATLs can dimerize in cis and trans, and are believed to tether adjacent membranes and bring them closely together to allow fusion. ATLs can mediate fusion in vitro, but for efficient fusion additional factors were proposed [14]. Mammalian ATLs differ in tissue expression, with ATL1 being mainly expressed in the CNS $[8,15]$ whereas ATL2 
and ATL3 are more ubiquitously expressed [16]. All ATLs localize to the ER, ATL1 throughout ER tubules, ATL2 and 3 more concentrated to three-way-junctions ( $3 \mathrm{WJs}$ ) [7, 11, 17].

A role for ATLs in membrane traffic is less clear. Two publications found no role for ATL1-3 in ER-Golgi transport, assayed by knock-down, KO or overexpression of dominant negative ATLs [16, 18]. Another study concluded that ATL1 plays a role in vesicle trafficking at the ER/Golgi interface, but transport was not directly assessed [19]. Recently, it was shown that knockdown of ATL2 but less so of ATL3 in HeLa cells reduced ER-Golgi transport [20].

The pathomechanism of ATL mutations thus remains largely unclear. ATLs are directly or indirectly involved in many ER-related cellular processes like trafficking [21], ER stress [18] and lipid droplet biogenesis [22]. Many findings support the notion that mutated ATLs are defective in forming a fully functional axonal ER (reviewed in Ref. [1, 3]). Interestingly, not all HSP or HSAN disease variants of ATLs are defective in ER network formation, GTP-hydrolysis, dimer formation and fusion [23].

We here report that the ATL3 disease variant Y192C slows down ER-Golgi trafficking, induces Golgi disruption, causes ER morphology defects, reduces autophagosome formation and affects nuclear shape.

\section{Materials and methods}

\section{Antibodies, plasmids and chemical compounds}

See supplementary experimental procedures.

\section{Cloning}

For construction of untagged human ATL3, ATL3 was amplified from hATL3-myc pCI neo [11] using forward (fwd) primer 5'-ggatccATGTTGTCCCCTCAGCGAGTGG3', introducing a BamHI site and reverse (rev) primer 5'-gcggccgcCTATTGAGCTTTTTTATCCATGGATGGTCTTCC$3^{\prime}$, introducing a stop codon and a NotI restriction site. For construction of N-terminal myc-tagged human ATL3, ATL3 was amplified from hATL3-myc pCI neo using primers fwd 5'-ggatccATGGAACAAAAACTTATTTCTGAAGAAGAT CTGTTGTCCCCTCAGCGAGTGG-3', introducing a N-terminal myc-tag and a BamHI site and rev: 5'-gcggccgcCTA TTGAGCTTTTTTATCCATGGATGGTCTTCC-3', introducing a stop codon and a NotI restriction site. In both cases, the restriction sites were used for cloning the respective fragment into pcDNA3.1 (+) Hygro. N-terminally GFP-tagged human ATL3 was constructed by amplifying ATL3 from hATL3-myc pCI neo using fwd primer 5'-CTCGAGCTA TGTTGTCCCCTCAGCGAGTGG-3', introducing a XhoI site, and rev primer 5'-ggatccCTATTGAGCTTTTTTATC CATGGATGGTCTTCC-3', introducing a stop codon and a BamHI restriction site. The restriction sites were used for cloning the fragment into pEGFP C1. The Y192C mutation was introduced by standard site-directed mutagenesis. All sequences were validated.

\section{Cell lines, cell culture, transfection}

HeLa Kyoto (in the following called HeLa) and $\mathrm{HuH} 7$ cells were maintained in Dulbecco's modified Eagle Medium + GlutaMax (Invitrogen) supplemented with 10\% FBS and incubated at $37{ }^{\circ} \mathrm{C}, 95 \%$ relative humidity and $5 \% \mathrm{CO}_{2}$. Cells were transfected using Lipofectamine 2000 according to manufacturer's instruction. Primary fibroblasts from a patient with the heterozygous Y192C mutation in ATL3 gene were derived from a 3-mm diameter punch biopsy. The procedures were approved by the local ethics committee (reference number A145/11) of the ChristianAlbrechts University Medical School (Kiel, Germany). Control fibroblasts 1.1 (AG13334) and 1.2 (AG04151) were obtained from the National Institute of Ageing collection of the Coriell Cell Repository (Camden) and are from the same donor at different age. Patient fibroblasts and controls were maintained in Dulbecco's modified Eagle Medium + GlutaMax (Invitrogen) supplemented with 20\% FBS and incubated at $37{ }^{\circ} \mathrm{C}, 95 \%$ relative humidity and $5 \% \mathrm{CO}_{2}$. Fibroblasts were immortalized by lentiviral infection with $\mathrm{pCDH}$ hTERT.

\section{Neuronal cell culture}

Cortical neurons were isolated from murine embryonic brains (E15.5) and maintained in glia-conditioned neurobasal medium. For transfection, the calcium-phosphate method was used as described [24] with the following changes: DNA-calcium phosphate-precipitates were prepared by mixing $80 \mathrm{ng} / \mu \mathrm{l}$ plasmid DNA and $250 \mathrm{mM} \mathrm{CaCl}_{2}$ with equal volumes of $2 \times \mathrm{BES}$ buffered saline. Coverslips with attached neurons were transferred to a dish containing transfection medium $(800 \mu \mathrm{M}$ sodium pyruvate, $8 \mathrm{mM}$ HEPES, $0.16 \%$ glucose, $5.5 \% \mathrm{ddH}_{2} \mathrm{O}$ in MEM, pH 7.65). Subsequently, neurons were incubated at $37^{\circ} \mathrm{C}$ for $1 \mathrm{~h} 30 \mathrm{~min}$. Afterwards, neurons were incubated for $10 \mathrm{~min}$ in washing medium ( $800 \mu \mathrm{M}$ sodium pyruvate, $8 \mathrm{mM}$ HEPES, $0.16 \%$ glucose, $5.5 \% \mathrm{ddH}_{2} \mathrm{O}$ in MEM, $\mathrm{pH} 7.35$ ) and moved back to their original dish containing glia-conditioned medium. After $24 \mathrm{~h}$, neurons were processed for immunocytochemistry.

\section{Immunocytochemistry and microscopy of cells}

For immunofluorescence cells were grown on coverslips. After incubation, cells were fixed with $4 \%$ paraformaldehyde 
and processed for immunofluorescence as described [25] with antibodies as indicated. For staining of RTN4, cells were fixed with $4 \%$ paraformaldehyde containing $0.1 \%$ glutaraldehyde as described [26]. For Fig. S1a cells were fixed with $3 \%$ glyoxal at pH 5 as described [27]. For Fig. S2a cells were fixed with ice-cold $\mathrm{MeOH}$ for $20 \mathrm{~min}$ at $-20{ }^{\circ} \mathrm{C}$. Nuclei were stained with Hoechst 33342 (Invitrogen H1399). Images were acquired on a Zeiss Axiovert200 using $20 \times$, $40 \times$ or $63 \times$ objective and Zen 2012 software. For some settings a confocal-like Apotome slider was used. Images were assembled and processed in Adobe Photoshop. Care was taken that identical settings were applied where images were to be compared. Weak signals like small vesicles or thin ER tubules were enhanced using gamma settings $<1$ to match the image perception by eye. For live cell imaging, cells were plated on $3.5-\mathrm{cm}$ glass bottom microwell dishes and imaged in DMEM without phenol red.

\section{Quantification of immunocytochemistry}

The number of fluorescently stained structures within cells was quantified using ImageJ software. For this purpose, images were converted to 8-bit grayscale pictures before converting to binary pictures to distinguish objects of interest from background. Overlapping objects were separated using the 'Watershed' function. Finally, distinct particles were counted using the 'Analyze particle' function. Y192Cexpressing $\mathrm{HuH} 7$ cells were smaller in size, therefore, $\alpha$-tubulin staining and CellProfiler were used to quantify and normalize cell size. For quantification of the Golgi fragmentation index, the relative frequency distribution of the number of fragments per cell for each condition was tabulated and the index was calculated using a published formula [28].

\section{Cell lysis, western blotting, deglycosylation assay}

Cells were lysed in STEN buffer, separated by SDS-PAGE, transferred to PVDF membranes and probed with antibodies as described [29]. Deglycosylation of VSVG-EYFP was performed basically as described [29] but using direct cell lysates instead of immunoprecipitates.

\section{Autophagy assay}

Cells seeded on cover slips were transfected with respective plasmids for $6 \mathrm{~h}$ followed by medium change. After $24 \mathrm{~h}$, cells were treated with control medium or pretreated with medium containing $50 \mu \mathrm{M}$ chloroquine (CQ) for $1 \mathrm{~h}$ followed by starvation medium [30] with $50 \mu \mathrm{M} C \mathrm{CQ}$ for $3 \mathrm{~h}$. Finally, cells were fixed and processed for immunocytochemistry.

\section{ER stress assay}

HeLa cells were seeded in 6 well plates and transfected the next day with respective plasmids for $6 \mathrm{~h}$, followed by medium change. $24 \mathrm{~h}$ after transfection, cells were treated with $2.5 \mu \mathrm{g} / \mathrm{ml}$ tunicamycin or DMSO for $24 \mathrm{~h}$, lysed and processed for western blotting.

\section{SEAP assay}

For measuring secretion activity HeLa cells stably expressing SEAP were used as described [31].

\section{Stain-free gel imaging and protein quantification}

Stain-free gel imaging was used to assess total protein levels (loading control). After separation, gels were soaked in $10 \%$ TCA solution for 5 min followed by three washing steps with $\mathrm{dH}_{2} \mathrm{O}$. Subsequently, gels were imaged using the Gel Doc System (Bio-Rad). The intensity of whole lanes was quantified after background subtraction using the Rolling Ball algorithm in ImageJ. The obtained intensity values were used to normalize the western blot signals of proteins of interest. As described by others [32,33] we found this method superior to normalization to actin or tubulin or other house-keeping genes.

\section{Statistics}

Reported values represent mean \pm SEM, unless stated otherwise. Statistical differences between the means of two groups were determined using Welch's $t$ test, unless stated otherwise. Numbers of independent replicates and $p$ values are indicated in figures or figure legends, a $p$ value $<0.05$ was considered significant.

\section{Results}

\section{ATL3 Y192C disrupts the ER network and deforms the nucleus}

ATL3 is expressed ubiquitously [16]. We, therefore, argued that general cellular functions of ATL3 can be analyzed in cell lines. To this end, we transfected myc-tagged ATL3 or the HSAN-causing mutation ATL3 Y192C in HeLa cells and analyzed their subcellular localization (Fig. 1a). As published before [11], ATL3 localizes predominantly to ER $3 \mathrm{WJ}$, whereas the Y192C variant is distributed along a misshaped ER with much less 3 WJs. Untagged as well as $\mathrm{N}$ - and C-terminally myc-tagged ATL3 localize to $3 \mathrm{WJ}$ (Figure S1a), confirming previous reports $[7,11,17]$ and demonstrating that the tag does not interfere with localization. 
Fig. 1 ATL3 Y192C induced ER-morphology changes and malformation of the nucleus. a HeLa cells were transfected with a control vector, ATL3myc or ATL3 Y192C-myc, fixed and stained with antibodies against calnexin and myc. Arrows indicate punctate ATL3 localization, arrowheads ATL3 Y192C mislocalization at distorted ER. b, d HeLa cells transfected as indicated were lysed and processed for immunoblotting with anti-ATL3 antibodies. The arrows indicate endogenous ATL3. Equal loading was validated by stain-free gel-imaging (see Fig. S6, also for full-size blots). $\mathbf{c}$ HeLa cells were transfected with pplssKDEL-mRFP (RFP-KDEL) as ER marker and GFP-ATL3 or GFP-ATL3 Y192C and analyzed by live cell imaging. Lower panel, magnifications of the boxed areas. e HeLa cells were transfected with a control vector, ATL3-myc or ATL3 Y192C-myc, fixed and stained with antibodies against myc and lamin A. Arrows point to distorted nuclei. a, c, e Single Apotome sections of representative images from $n=3$ independent experiments. Nuclei in a, c were stained with Hoechst 33342, scale bar $10 \mu \mathrm{m}$
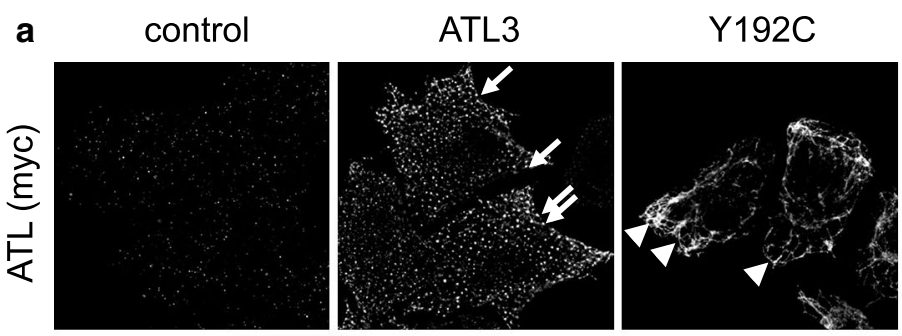

b
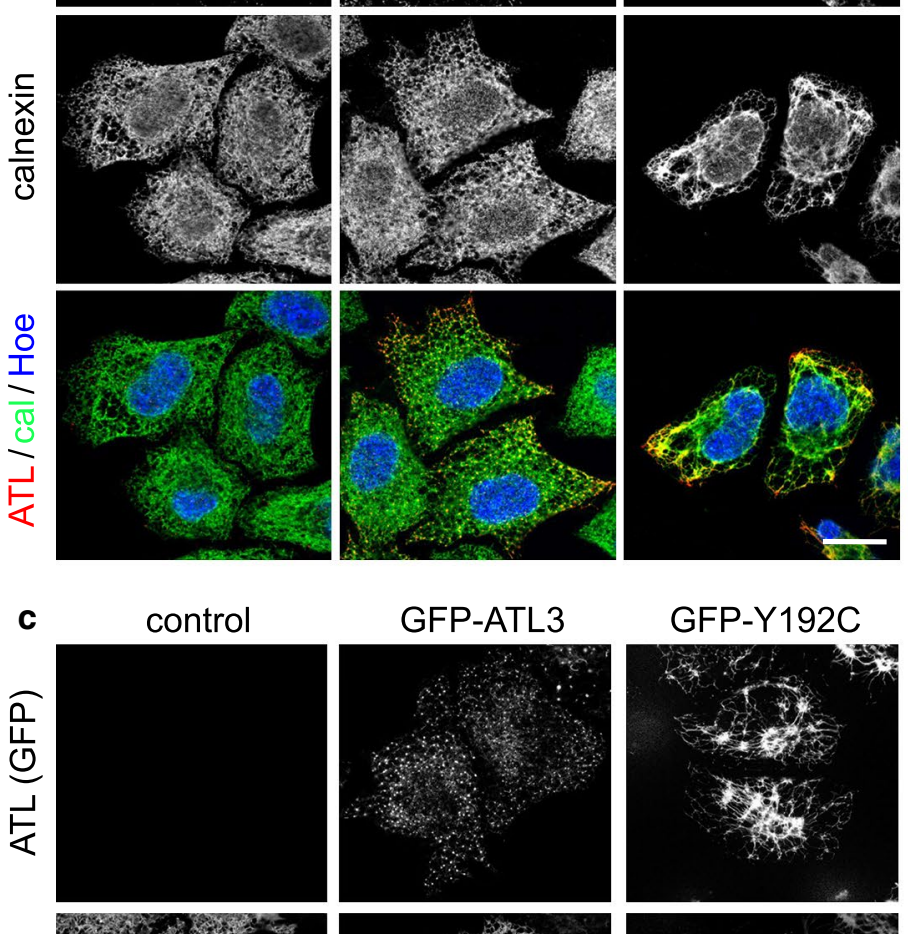

d
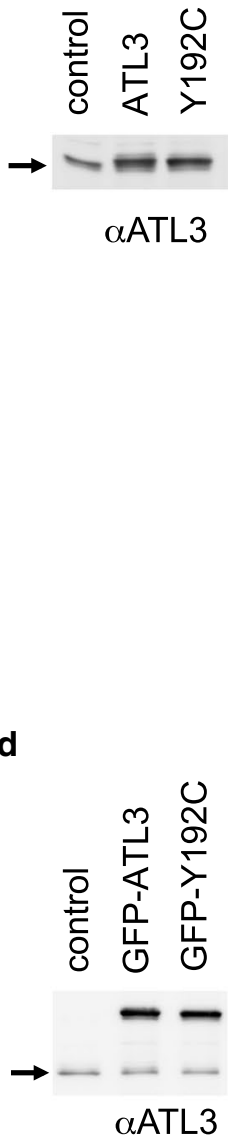
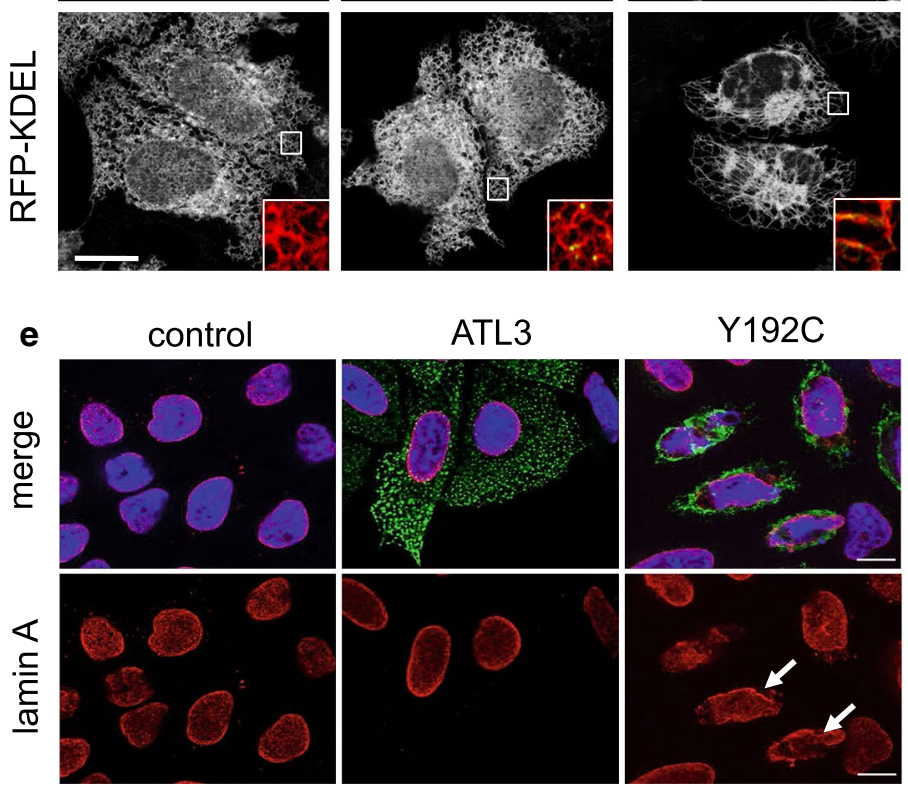

ATL3

Y192C
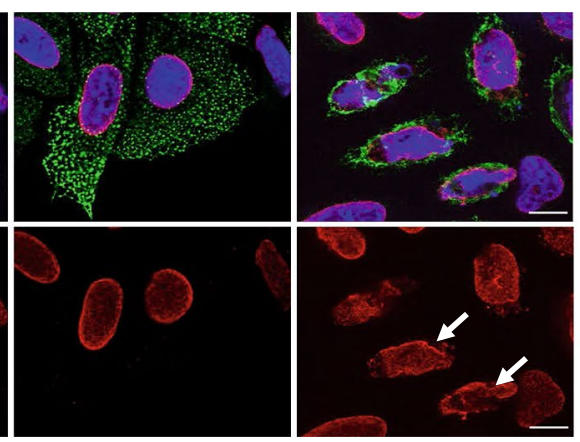

Expression levels of ATL3 and ATL3 Y 192C are comparable, suggesting that no differences in expression levels cause the different phenotypes (Fig. 1b). We next analyzed the ER morphology by live-cell microscopy using GFP-ATL3 variants (Fig. 1c). While ATL3 overexpression does not change the extensive tubular ER-network, the Y192C mutation had a strong impact on the ER. ER network complexity was strongly reduced with elongated tubules and fewer 3 WJs. The GFP-tag is tolerated only at the N-terminus, not the C-terminus of ATL3. ATL3-GFP does not localize to $3 \mathrm{WJs}$, 
but is more broadly distributed in the ER, suggesting that only small tags at the C-terminus are tolerated (Fig. S1c). A similar ER morphology change is induced by overexpression of the corresponding mutation in ATL1, Y196C (Fig. S2a). Overexpression of ATL3 Y192C did not affect the microtubule network (Fig. S2b). It did also not cause up-regulation of BIP, suggesting the overexpression of the mutant ATL3 does not cause ER stress (Fig. S2c, d). Interestingly, we noted a deformation of the nucleus in most ATL3 Y192Ctransfected cells ( $83 \%$ deformed nuclei \pm 0.04 vs. $8 \% \pm 0.03$ in ATL3-transfected cells; SEM, $n=3 ; 224$ and 369 cells, respectively; Fig. 1e).

\section{ATL3 Y192C delays ER export by reducing the number of ERES}

To test whether ATL3 Y192C affects export from the ER we made use of the temperature-sensitive tsO45 mutant VSVG [34] tagged with EYFP (VSVG-EYFP) [35]. This trafficking reporter is misfolded at $40{ }^{\circ} \mathrm{C}$ and consequently accumulates in the ER. Upon shifting to the permissive temperature $\left(32{ }^{\circ} \mathrm{C}\right)$, VSVG-EYFP folds properly, exits the ER and travels to the plasma membrane [36, 37]. Using its $\mathrm{N}$-glycosylation sites the localization can be assessed with the help of endoglycosidaseH (endoH), because ER-localized VSVG-EYFP is endoH-sensitive whereas Golgi and beyond-localized VSVG-EYFP is endoH resistant [31]. In HeLa cells transfected with ATL3 or ATL3 Y192C together with VSVG-EYFP only the Y192C mutation, but not the wild type, slowed down ER-Golgi transport (Fig. 2a-c). Overexpression of the dominant-negative Sar1 H79G mutant [38] served as positive control for transport inhibition. Linear regression analysis and slope determination indicated that ATL3 Y192C expression compared to ATL3 slowed down the acquirement of endoH resistance by app. $25 \%$ (slope: control 0.017 \pm 0.0003 ; Sar1 H79G 0.005 \pm 0.0008 ; ATL3 0.016 \pm 0.0005 ; ATL3 Y192C $0.012 \pm 0.0006)$. The findings were corroborated by measuring the secretion rate of a secreted alkaline phosphatase (SEAP) [39], which was also reduced in ATL3 Y192C expressing cells compared to ATL3 (Fig. 2d). BFA [40] served as positive control. The data suggest that ATLs are directly or indirectly involved in ER export, possibly via their influence on ER morphology.

To analyze the underlying cause for this reduced export capacities we probed cell lysates for the presence of Sec23 and Sec31, two COPII components of the molecular machinery involved in ER export [41]. No difference in expression levels of both was observed in ATL3 or ATL3 Y192C expressing cells (Fig. 3a). However, in ATL3 Y192C expressing cells the number of ER exit sites (ERES) as identified with two different ERES-components, Sec31 (Fig. 3b, c; Fig. S4) and Sec16 (Fig. S3b, c, Fig. S4) was reduced by around $45 \%$. The reduced ER export rate could affect the ER-Golgi intermediate compartment (ERGIC), a very dynamic organelle between the ER and the Golgi (reviewed in Refs. [42, 43]). Indeed, ATL3 Y192C but not ATL3 transfection in Hela cells resulted in reduced ERGIC53 staining (Fig. S3a). ERGIC53 is a marker of the ERGIC [44]. In addition, the typical ERGIC distribution in a Golgi-like, juxtanuclear accumulation and numerous peripheral vesicles was transformed to a broader distribution partially colocalizing with the distorted ER and loss of the peripheral vesicles (Fig. S3a). This suggested a disturbed recycling of ERGIC components and partial retention in the ER due to the reduced ER-export. We next analyzed the Golgi complex, because reduced transport to the Golgi may result in morphological changes like fragmentation, often observed in neurodegenerative diseases (reviewed in Refs. [45, 46]). Indeed, expression of ATL3 Y192C, but not ATL3 resulted in a fragmentation of the Golgi, as indicated by staining the Golgi with an antibody against giantin (Fig. 3d) and by determining a Golgi fragmentation index (Fig. 3e). The Golgi fragmentation is not caused by a disturbed microtubule network (Fig. S2b). Expression levels of two Golgi proteins, GM130 and giantin, remained unaffected (Fig. 3f).

Taken together, the data suggest that by reducing the number of ERES, ATL3 Y192C causes a reduced transport of cargo from the ER, resulting in disruption of the ERGIC and fragmentation of the Golgi.

\section{ATL3 Y192C reduces the formation of autophagosomes}

The ER plays an important role in autophagy, and ERES are key players in autophagosome formation (reviewed in Ref. [47]). We, therefore, studied whether ATL3 Y192C may affect autophagy by quantifying the number of LC3positive structures. LC3 in its lipidated form is a marker for autophagosomes [48]. Expression of ATL3 Y192C but not ATL3 in HuH7 cells significantly reduced the number of LC3-positive structures in untreated as well as in starved cells treated with chloroquine (Fig. 4a, b). This suggests that the ER morphology changes and/or the reduced ERES number induced by ATL3 Y192C severely affects autophagosome formation or processing.

\section{ATL3 Y192C is excluded from axonal ER}

We next wanted to analyze the ATL3 Y192C mutation in neurons. To this end, we transfected ATL3 or ATL3 Y192C in DIV1 (1 day in vitro) murine cultured cortical neurons and analyzed them $24 \mathrm{~h}$ later (DIV2). In DIV2 neurons ATL3 is distributed throughout the longest neurite (the future axon) and the shorter neurites, whereas ATL3 Y192C is mostly restricted to the cell body and not extending into neurites (Fig. 5a). The overexpression of ATL3 Y192C strongly 


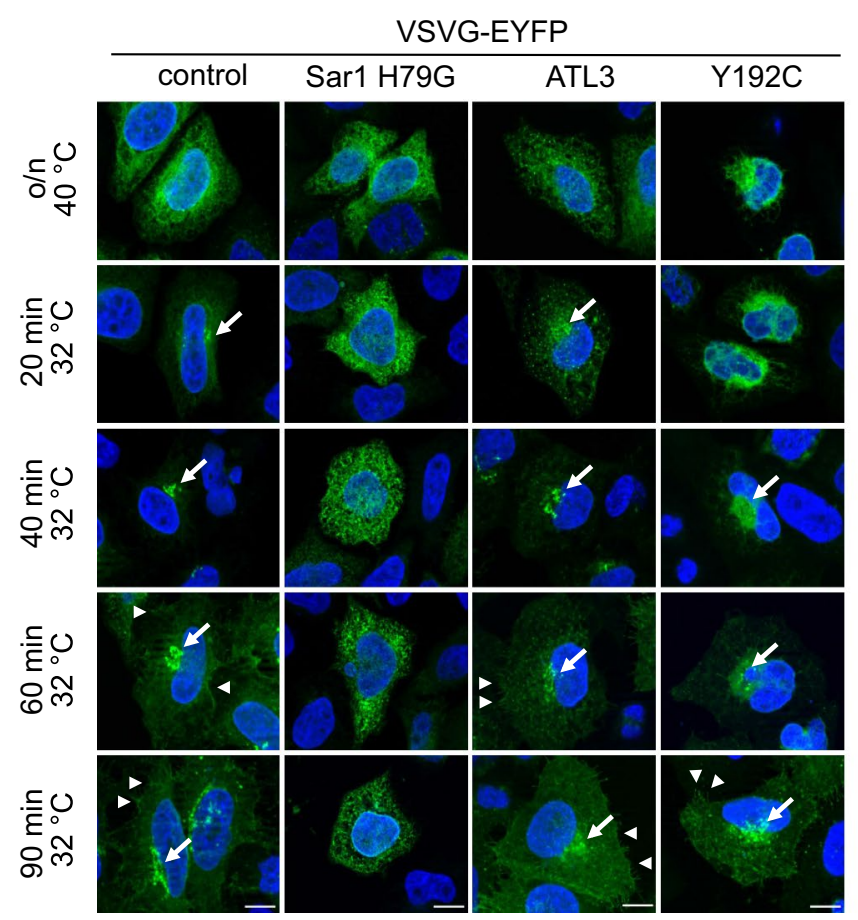

b

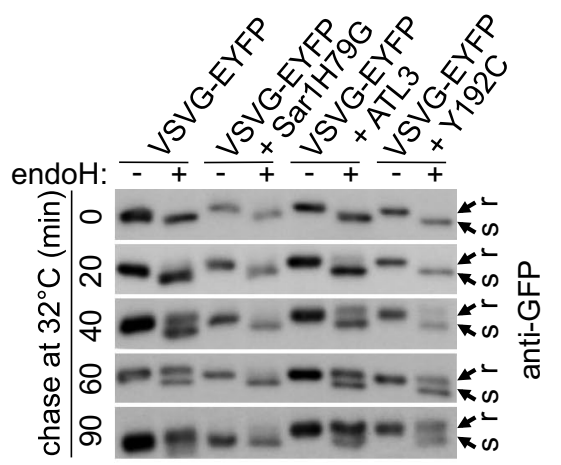

C
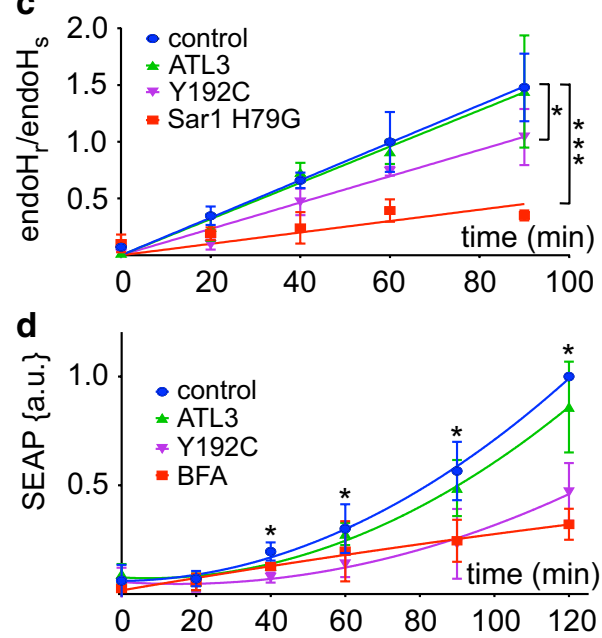

Fig. 2 ATL3 Y192C delayed ER to Golgi transport. HeLa cells were transfected with VSVG-EYFP and a control vector, Sar1 H79G, ATL3-myc or ATL3 Y192C-myc, incubated overnight at $40{ }^{\circ} \mathrm{C}$ and chased at $32{ }^{\circ} \mathrm{C}$ for indicated times, followed by fixation (a) or cell lysis (b). a Fixed cells were stained with an antibody against myc to identify transfected cells. Nuclei were stained with Hoechst 33342. Images represent single Apotome sections. Arrows indicate Golgi localization of VSVG-EYFP, arrowheads VSVG-EYFP localization at the plasma membrane. For clarity only EYFP staining is shown. Scale bar, $10 \mu \mathrm{m}, n=3$ independent experiments. b Lysates were digested with or without endoH, separated using SDS-PAGE, blotted and probed with an antibody against GFP. Stain-free gel imaging was used as loading control (Fig. S6, see also for full-size blots). c Quan-

impairs the outgrowth of the future axon, reducing the mean length of the longest neurite by $50 \%$ (Fig. 5b). Interestingly, in ATL3 Y192C expressing cells the axon still contains ER as indicated by a fluorescent ER marker, tomato-KDEL (Fig. 5c, d) or endogenous RTN4 (not shown), suggesting a localization/transport defect of mutant ATL3 but not a collapse of the axonal ER. To test if this effect would be also observed in older, more mature neurons with already established long axons, we transfected DIV7 cultured neurons with ATL3 wt and Y192C mutant and tomato-KDEL (Fig. 5e, f). In ATL3 expressing neurons the ATL3 staining extends into the axon almost as far as the ER marker. In contrast, in ATL3 Y192C expressing neurons the mutant ATL3 only extends into proximal axon segments, whereas tification of the ratio of the upper, endoH resistant and the lower, endoH sensitive band. Signals were quantified using ImageJ. Data points were fitted using GraphPad Prism's linear regression function. Data are displayed as mean $\pm \mathrm{SD}, * p<0.05$ (one-way ANOVA followed by Tukey's multiple comparisons test, slopes of each linear regression function were compared). $n=3$ independent experiments. d HeLa cells stably expressing SEAP were transfected with a control vector or ATL3-myc or ATL3 Y192C-myc or treated with BFA $(0.1 \mu \mathrm{g} / \mathrm{ml})$. After the indicated times, SEAP activity was determined, data normalized against the obtained maximum and minimum value and fitted using GraphPad Prism's polynomial second order function. Data are displayed as mean $\pm \mathrm{SD}, * p<0.05$, ***p $p<0.001$, Welch's $t$ test, $n=4$ independent experiments

tomato-KDEL also is present in distal axons (Fig. 5f). This suggests that mutant ATL3 Y192C cannot properly localize to the axonal ER and that this lack of axonal ATL3 contributes to disease pathology.

\section{ATL3 Y192C patient cells display a reduced ER network complexity and are compromised in autophagy}

To analyze the effect of ATL3 mutations under pathophysiological conditions, we studied immortalized fibroblasts from an individual harboring the Y192C mutation and healthy controls (Fig. 6). Compared to two fibroblast cell lines derived at different age from a healthy donor, the 
a

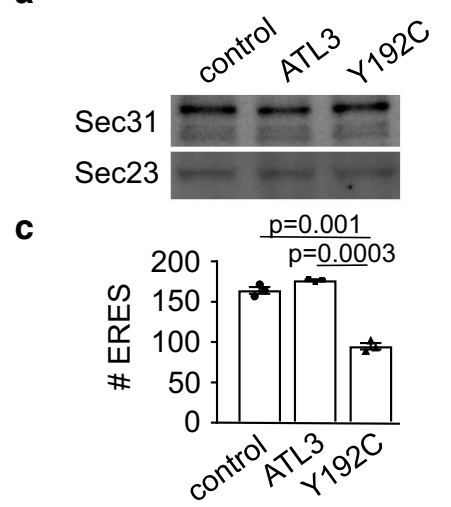

b

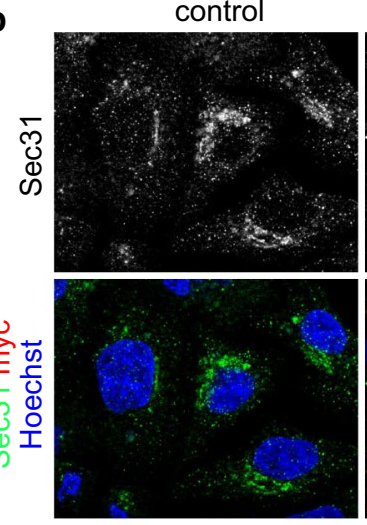

ATL3
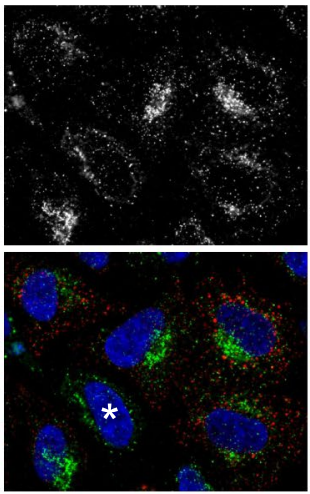

Y192C

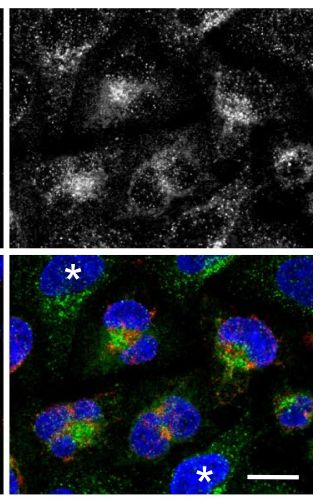

d
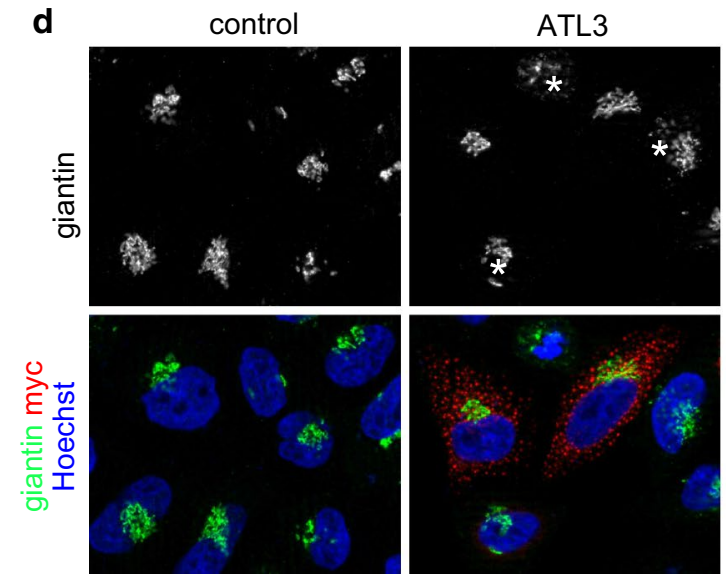

Fig. 3 ATL3 Y192C reduced the number of ERES and fragmented the Golgi. HeLa cells were transfected with a control vector or ATL3myc or ATL3 Y192C-myc, incubated for $24 \mathrm{~h}$, lysed (a, f) or fixed and processed for immunofluorescence with indicated antibodies and Hoechst 33342 to label nuclei (b, d). a Lysates were blotted and probed with antibodies against $\operatorname{Sec} 31$ and Sec23. $n=3$ independent experiments. b Single Apotome sections of transfected HeLa cells as indicated. Representative images of 3 independent experiments are shown, scale bar, $10 \mu \mathrm{m}$. c Quantification of $\mathbf{b}$. Sec31 labeled ERES were counted using ImageJ, for example images see Fig. S4.

patient fibroblasts have a less complex tubular ER network (Fig. 6a). For investigation of autophagic vesicles two additional fibroblast lines from healthy donors were compared to the patient cell line. Corroborating the results in transfected HeLa cells, the number of steady-state as well as starvationinduced LC3-positive vesicles was significantly reduced in patient cells compared to all four controls (Fig. 6b). p62/ sequestosome 1 is an autophagy receptor that is degraded in autophagic conditions and increased upon inhibition of autophagy [49]. Consequently, upon autophagy stimulation p62 levels were reduced in the four control fibroblast lines, but increased in ATL3 Y192C fibroblasts, indicating a compromised autophagy (Fig. 6c, d). All fibroblast lines expressed similar levels of ATL1-3 (Fig. S5). Taken together, also patient cells show a compromised ER and defects in autophagy, confirming that the results obtained
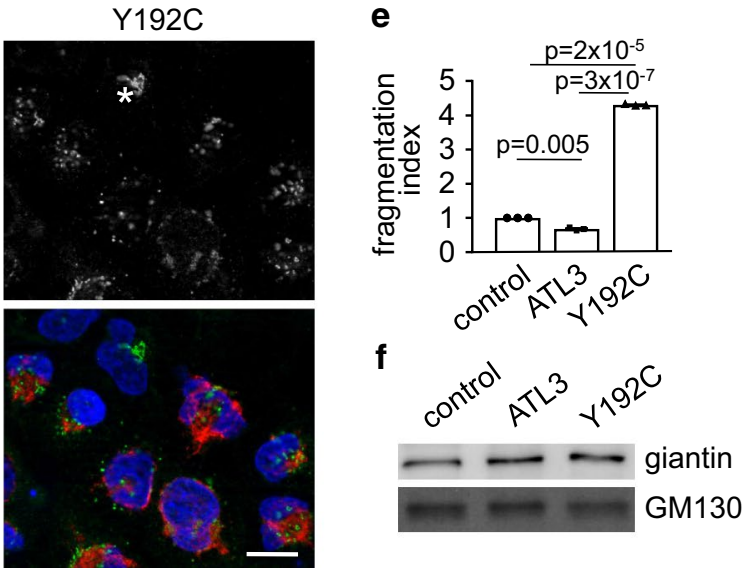

Analyzed were 66-120 cells per condition from $n=3$ independent experiments. d Transfected cells were stained with antibodies against giantin and myc. b, d Asterisks indicate untransfected cells, scale bar $10 \mu \mathrm{m}$. e Quantification of d. Golgi fragments were counted using ImageJ and the fragmentation index calculated. Analyzed were 115 cells per condition from three independent experiments. f Lysates were blotted and probed with antibodies against giantin and GM130. $n=3$ independent experiments. a, f For full size blots and stain-free gel imaging as loading control see Fig. S6. c, e Values represent mean \pm SEM, $p$ values are indicated (Welch's $t$ test)

by overexpression mimic, albeit exaggerate, the patient situation.

In sum, the data suggest that ATL3 Y192C causes numerous defects in ER and Golgi morphology, ER-exit, autophagy and neurite outgrowth.

\section{Discussion}

Causative heterozygous mutations for HSP (SPG3A) and HSAN were identified in ATL1 and ATL3, respectively $[8,11,12]$. Both ATL1 and ATL3 are ER-shaping membrane proteins, but the precise pathomechanism has not been elucidated. We here show that the HSAN-causing ATL3 mutation Y192C but not ATL3 affects multiple ER-related pathways when overexpressed in cell lines or 
Fig. 4 ATL3 Y192C affects autophagosome formation. HuH7 cells transfected with empty vector (control), ATL3myc or ATL3 Y192C-myc were incubated for $24 \mathrm{~h}$, treated without (steady state) or with starvation medium plus $50 \mu \mathrm{M}$ chloroquine (starv. + CQ) for $3 \mathrm{~h}$, fixed and processed for immunofluorescence staining with antibodies against LC3 and myc and with Hoechst 33342 to label nuclei. a Single Apotome sections of representative cells from $n=3$ independent experiments. Arrows, LC3-positive vesicles. Scale bar $10 \mu \mathrm{m}$. Note that the starvation medium causes ER morphology changes, indicated by the relocalization of ATL3 to larger aggregates (arrowheads). b Quantification of cells from a. Displayed is the average number of LC3-positive vesicles per cell normalized to the cell size. Error bars depict SEM. Analyzed were 26-30 cells for each condition from three independent experiments, $p$ values are indicated (Welch's $t$ test) a
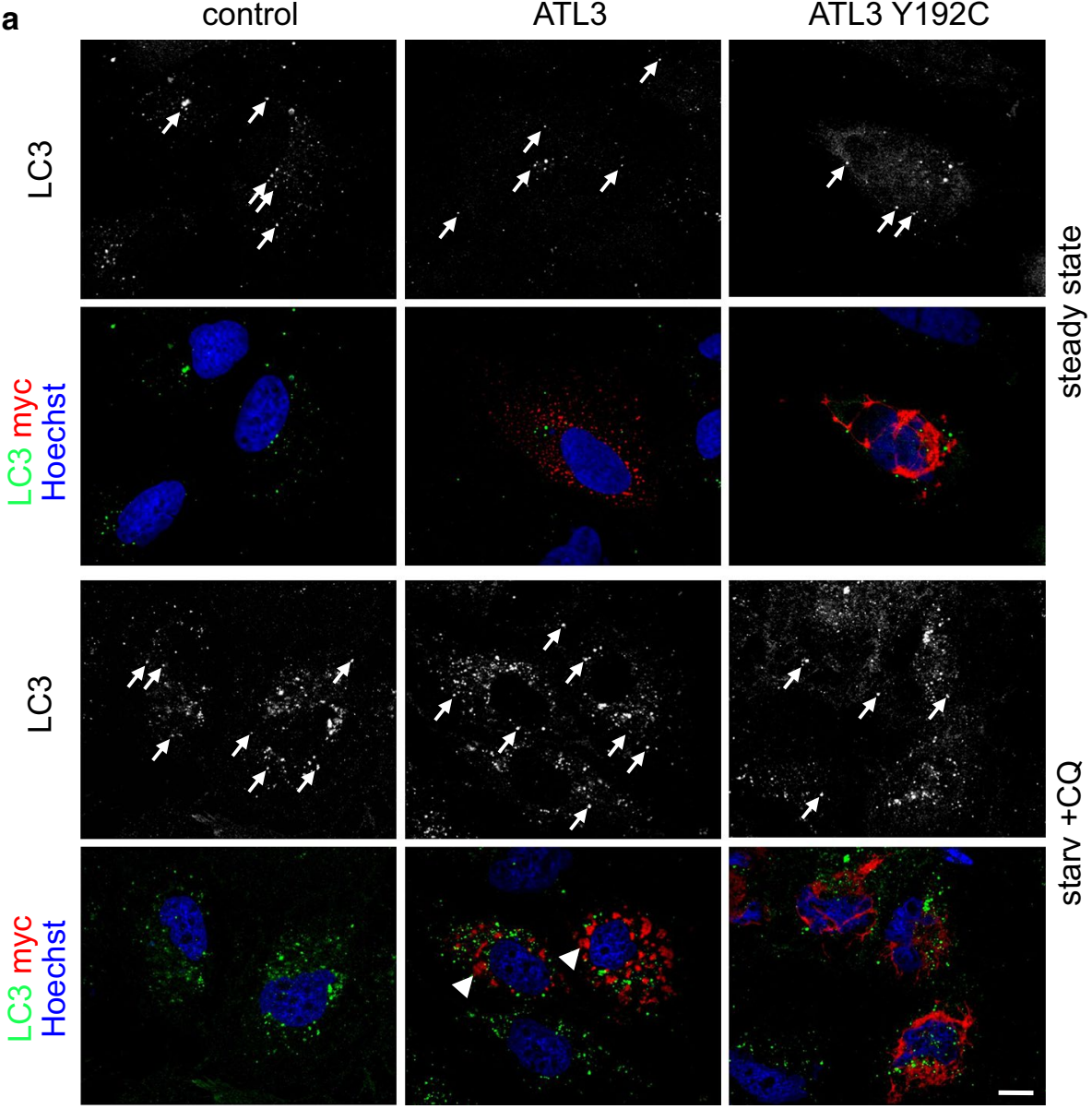

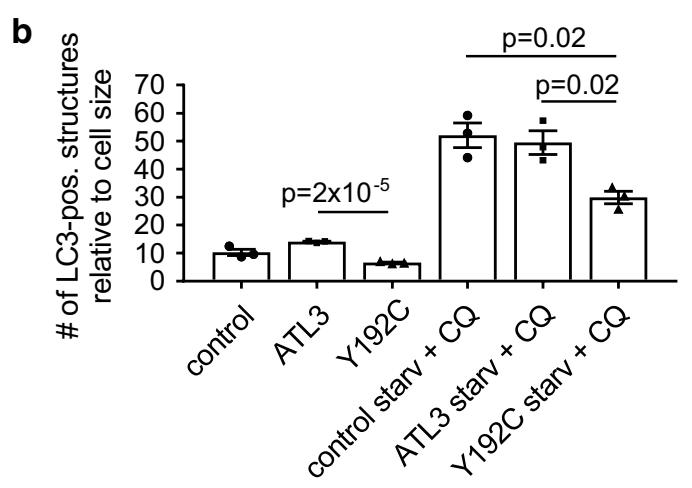

cultured neurons. The ATL3 Y192C is a dominant negative mutation [11], justifying the use of an overexpression paradigm to study the role of the mutation, although this does not fully match the patient situation. However, since in all our assays only the overexpression of ATL3 Y192C but not ATL3 caused a phenotype we are confident that the observed effects are indeed due to the mutation and not the overexpression per se. In addition, using fibroblasts from a HSAN patient carrying the Y192C mutation heterozygously, we could confirm that features we detected by transfection (ER morphology, autophagy defects) are similarly observed in the heterozygous patient cells. The overexpression approach most likely allows detection of cellular dysfunctions that may go unnoticed in patient fibroblasts in vitro. Subtle changes in, for example, ER-Golgi transport might be difficult to detect, but still be relevant for disease progression. The age of onset of HSAN1 caused by ATL3 Y192C is 14-30 years and only sensory neurons are affected [11], despite the wide-spread expression of ATL3. This indicates that cellular dysfunctions are subtle in patients and may need to be exaggerated in in vitro studies to detect them, but it should be kept in 
Fig. 5 Axonal outgrowth is inhibited and ATL3 Y192C is mislocalized in cultured neurons. Cortical murine primary neurons were transfected with myc-tagged ATL3 variants $(\mathbf{a}, \mathbf{b})$ or additionally cotransfected with tomato-KDEL as ER-marker (c-f), fixed $24 \mathrm{~h}$ later and stained with antibodies as indicated. The DIV at day of fixation is indicated. Arrows indicate axons. a Note the untransfected neuron just below the transfected one with a normally grown axon (asterisk). b Quantification of a. Displayed is the mean length of the longest neurite (the future axon). Error bars depict SEM. d, f Quantification from c, e. The ratio depicts the mean length of ATL3 staining divided by the mean length of axonal ER staining staining extending into the axon. a-f Analyzed were 32-51 neurons for each condition from three independent experiments. Error bars depict SEM, $p$ values are indicated (Welch's $t$ test).

Scale bar $10 \mu \mathrm{m}$
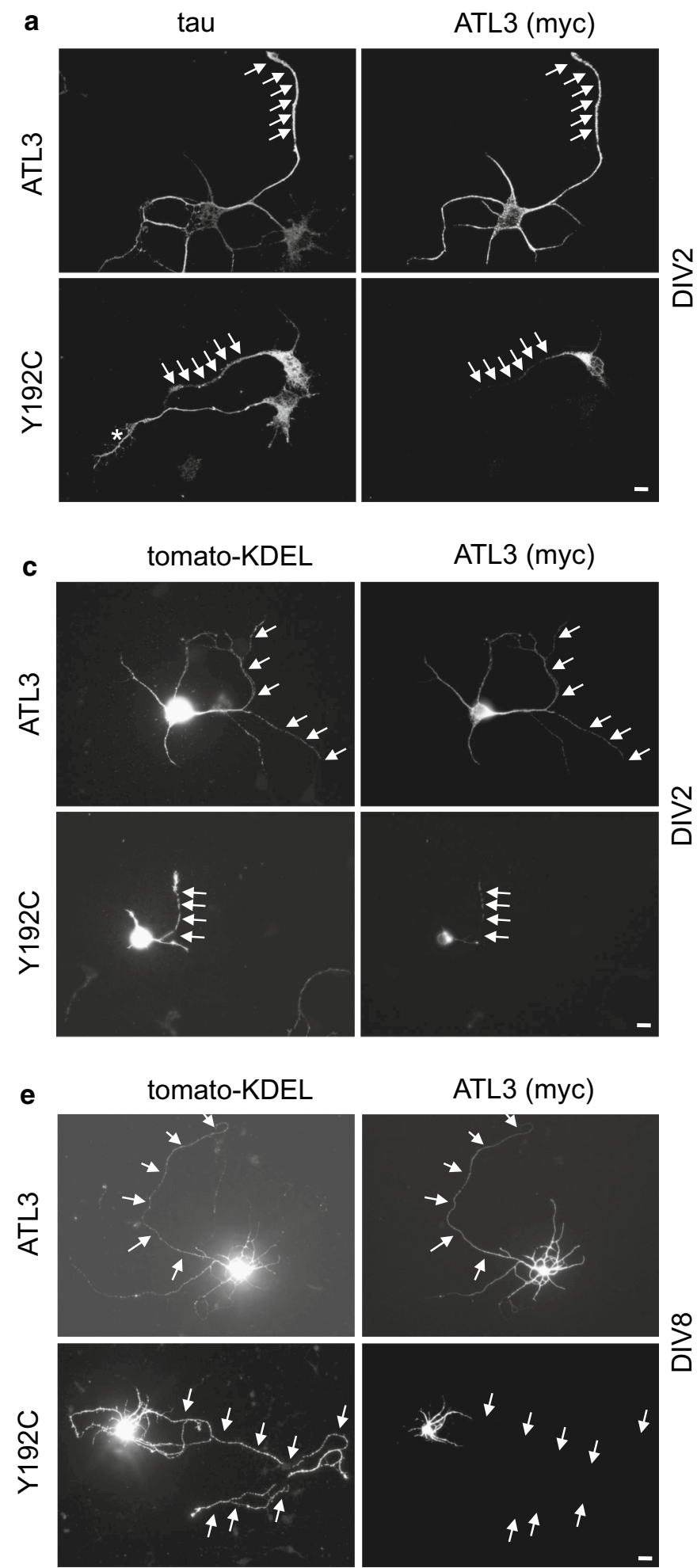

b
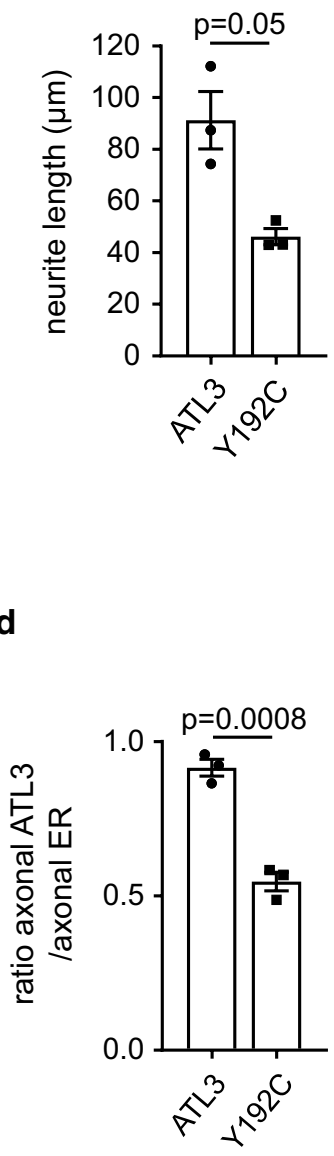

f

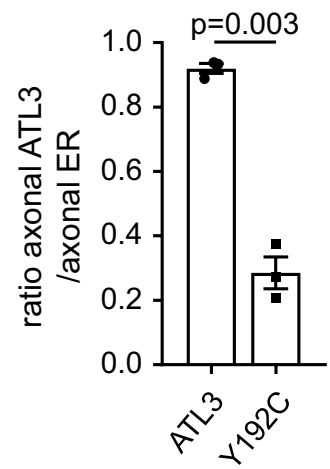

mind that the overexpression does not exactly mimic the patient situation.

Why are neurons with their extremely long axons preferentially affected? We speculate that their proteostasis systems might be simply already at their limits without further reserves, just coping with the load at young age.
Any further burden imposed by aging of the neuron in conjunction with the mutation then may lead to neurodegeneration. Less polarized cells may have more reserves (and in fact in most cases a much shorter lifespan) and therefore, remain asymptomatic. 
a
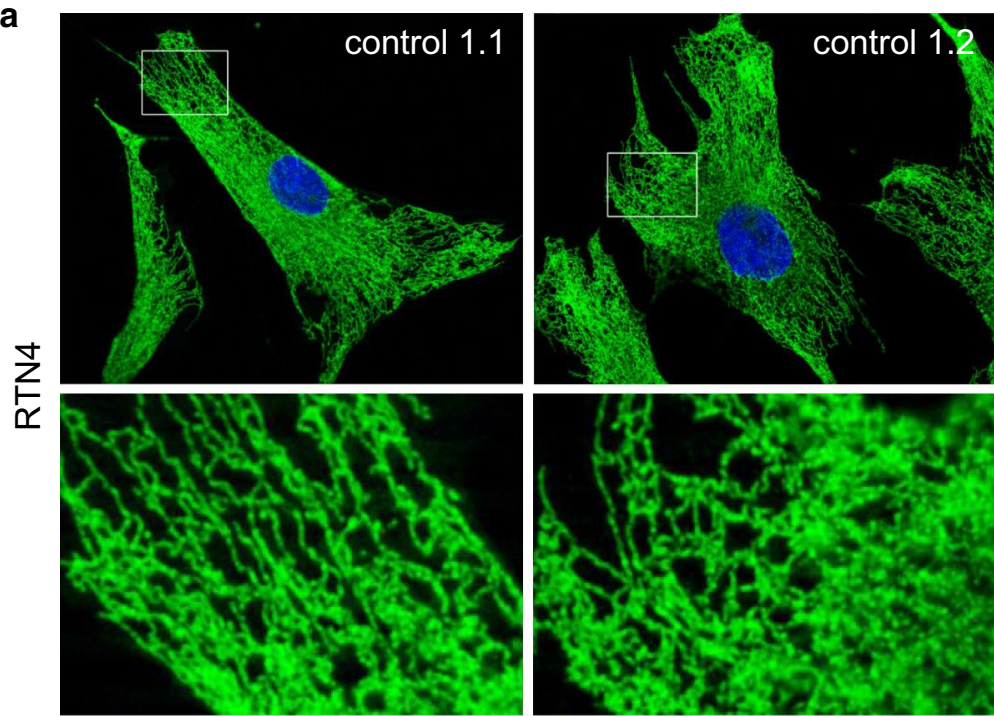

b

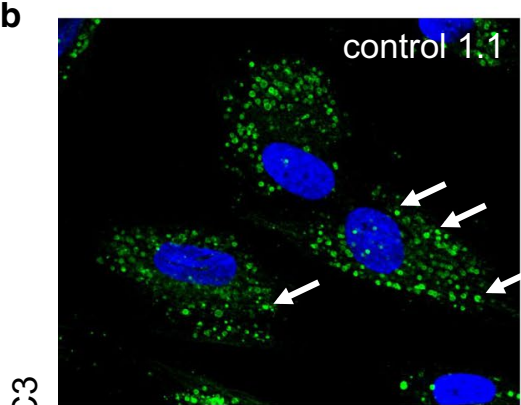

0
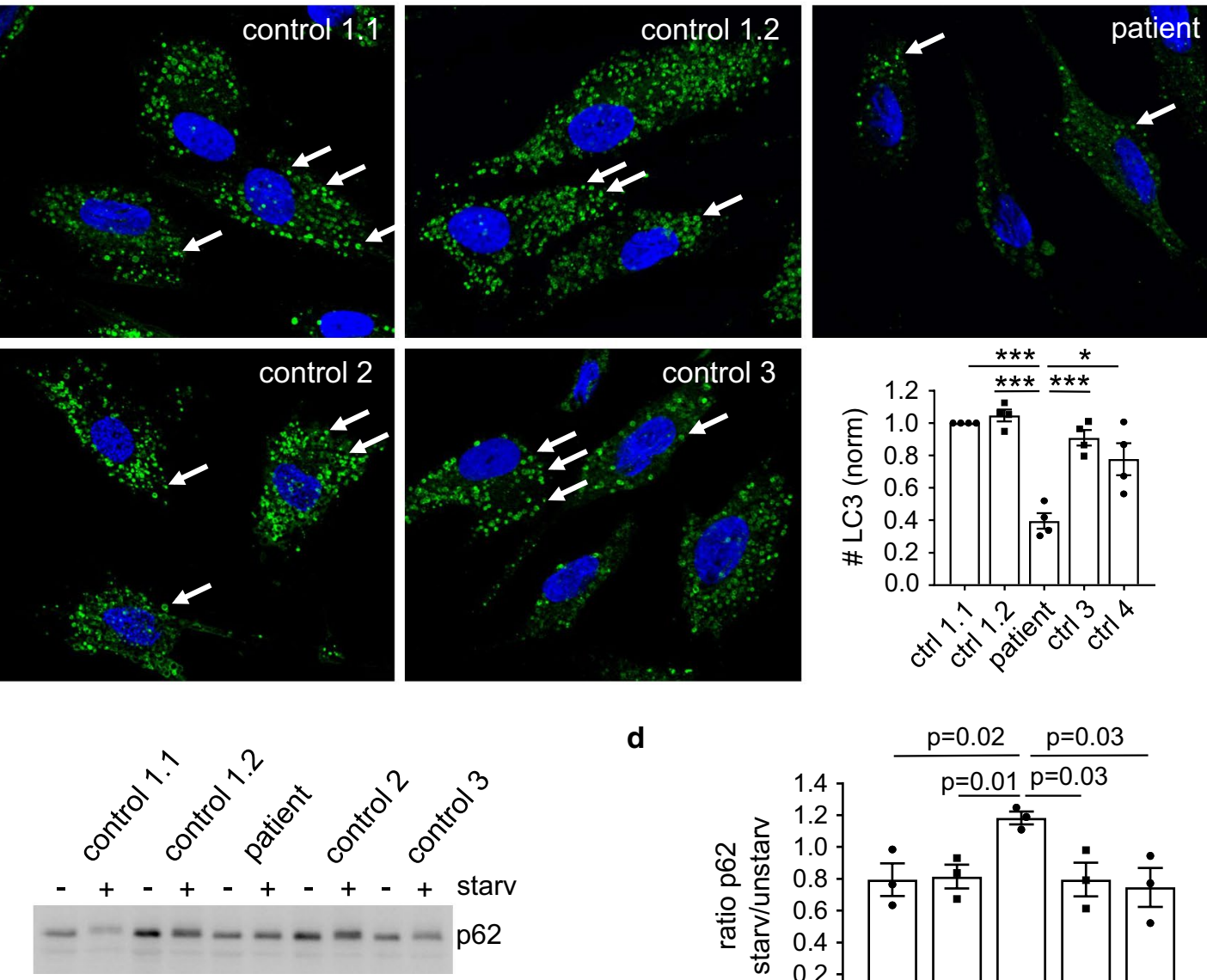

d

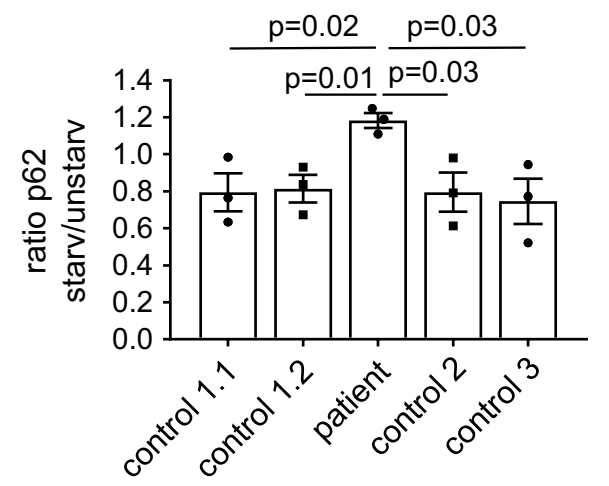

Using sensitive assays for ER-Golgi transport (with VSVG-EYPF) and secretion (with SEAP) we detected effects of the disease-causing ATL3 Y192C variant on ER

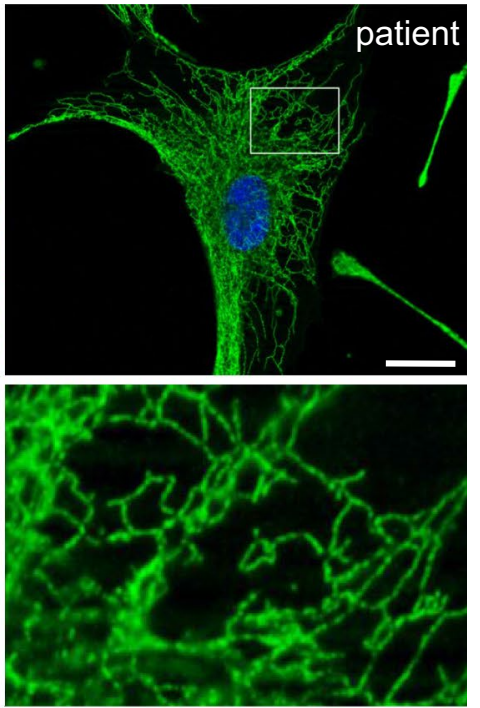

. 
4Fig. 6 Reduced ER complexity and deficits in autophagy in patient cells. Fibroblasts derived from healthy donors (control) and a HSAN1 patient with ATL3 Y192C mutation (patient) were incubated for $24 \mathrm{~h}$ and a fixed and processed for immunofluorescence staining with antibodies against RTN4 or $\mathbf{b}$ treated with starvation medium plus $50 \mu \mathrm{M}$ chloroquine (starv. $+\mathrm{CQ}$ ) for $3 \mathrm{~h}$. Thereafter cells were fixed and processed for immunofluorescence staining with LC3 antibodies and with Hoechst 33342 to label nuclei. Arrows, LC3-positive vesicles. Graph: displayed is the ratio of LC3-positive vesicles per cell normalized to control 1.1. Error bars depict SEM, ${ }^{*} p=0.001$; $* * * p=2 \times 10^{-5}$ to $2 \times 10^{-4}$ (Student's $t$ test). At least $177-239$ cells were analyzed for each condition from $n=4$ independent experiments. c Control and patient fibroblasts were treated with or without starvation medium for $3 \mathrm{~h}$, lysed, blotted and probed for p62. For full size blots and stain-free gel imaging as loading control see Fig. S6. d Quantification of $n=3$ independent experiments from c. Displayed is the mean ratio of p62 intensity in starved/unstarved conditions. Error bars depict SEM. $p$ values are indicated (Student's $t$ test). Single Apotome sections, scale bar $10 \mu \mathrm{m}$

the reduced ER-export. Indeed, ERGIC53, a marker for the ERGIC, seems to accumulate in the ER due to its reduced export. In contrast, using overexpression of dominant-negative ATL1-3, Rismanchi et al. found no effect on transport of VSVG-GFP to the plasma membrane [16]. However, the transport was not quantified and small changes might have gone unnoticed. Namekawa et al. indirectly concluded from changes in ER/Golgi morphology that ATL1 HSP-mutations affected trafficking, but no transport was analyzed [19]. In addition to the transport defects we observed a strongly compromised ER-network, reduced number of autophagosomes and increased p62-levels, defects in nuclear shape and neurite outgrowth deficits in primary neurons.

Are all the observed cellular changes directly caused by ATL3 Y192C, or are they indirectly the consequence of a single primary deficit? This is difficult to answer at this point. ATLs have mainly been associated with ERnetwork formation [5, 6] and a convincing model how ATLs mediates fusion was published [50]. The ATL3 Y192C mutation also causes severe deficits in establishing an ER network, up to total loss of tubules in highly overexpressing cells (this work, [11]). While this manuscript was in preparation, it was reported that the ATL3 Y192C mutation is defective in ER fusion and results in aberrant tethering of ER tubules [13]. It is conceivable that for proper assembly and function ERES need an exactly defined ER structure with the right degree of curvature and sheet/tubule ratio [51]. A condensed, non-branched ER induced by ATL3 Y192C would simply not provide enough suitable assembly sites for ERES components, resulting in reduced ER-export. The reduced numbers of ERES could also explain the deficits in autophagy, since ERES are tightly linked to the formation of autophagosomes [52, 53], and the axonal outgrowth deficits. Our findings on autophagy are in contrast to a recent report in which increased autophagy flux was observed in HeLa cells expressing ATL3 Y192C, while patient cells were not analyzed [54]. This discrepancy remains unclear and may be attributed to different cellular systems or levels of overexpression. Finally, the nuclear shape malformations we detected might be indirectly induced by the condensed juxtanuclear ER. Interestingly, ATLs via their function in maintaining a proper ER topology, sustain the efficient targeting of proteins to the inner nuclear membrane [20]. Maybe the nuclear malformations are a consequence of compromised transport to the inner nuclear membrane.

On the other hand, it is conceivable that ATLs mediate not only the fusion of ER tubules but have additional direct functions in the pathways affected here. This is supported by the study of Ulengin et al. who analyzed many ATL1 HSP-mutations and categorized them in two classes: one class comprised mutations with a reduced GTPase activity that had deficits in dimerization and membrane fusion, the other one mutations that had no obvious effect in the respective assays [23]. Additional functions of ATLs could encompass a direct role in ERES formation and/or a direct role in autophagosome formation.

ATL3 Y192C caused neurite growth defects in young neurons and interestingly was not present in distal axons in DIV2 and DIV8 cultured neurons. This cannot be explained by non-establishment or retraction of distal axonal ER or a non-continuous ER. Soluble KDELtagged ER-marker is distributed throughout the axon in ATL3 Y192C expressing neurons, indicating a continuous ER ranging into distal axons. It remains to be shown to what extend the ultrastructure of the ATL3-free axonal ER in ATL3 Y192C expressing neurons is changed and to what extend this contributes to pathology. How the mutant ATL3 is prevented from localizing to distal axons remains an open question, maybe involving unknown immobilization/segregation mechanisms.

Why does a mutation in a homologous position (Y196C in ATL1, Y192C in ATL3) of two very conserved proteins cause different diseases? One explanation could be different expression patterns, with ATL1 being more expressed in motor neurons and ATL3 in sensory neurons. Another explanation could be that ATLs have overlapping, but not identical functions. ATL3 is more concentrated in $3 \mathrm{WJ}$, whereas ATL1 is more evenly distributed along the ER $[7,11,17]$. This was suggested to be the consequence of a slower GTPase activity of ATL3 [17], pointing to differences between the molecules. Endogenous ATLs do not seem to form heterodimers [16], but if this is true for disease-causing mutated proteins in patient cells has to be investigated. Single or double knock-down/knock-out of one or two of ATL1-3 has little effect on ER morphology [18, 20]. Does this suggest that ATL1 Y196C and ATL3 Y192C are forming heterodimers with other ATL family members, explaining why they act dominant-negatively? During revision of 
this manuscript Liang et al. demonstrated that exogenously expressed ATL2 interacted with endogenous ATL3 [55], but further work is needed to fully address this point.

Future studies will also tell if one or more of the observed cellular deficits are causative for the axonal degeneration. A careful assessment and comparison of mutations in ATL1 and 3 but also of mutations in other ER-shaping HSP- or HSAN-genes with as many assays as possible might reveal commonalities or differences that point to the cellular pathway(s) whose malfunctions are actually responsible for the disease.

Acknowledgements We are grateful to Eric Snapp, Bertrand Kleizen, Cagatay Günes and Rainer Pepperkok for providing cDNA or antibodies. We thank the FLI facilities Imaging, Functional Genomics and Animal Facility. Special thanks to Daniela Reichenbach for neuronal cultures and Christina Valkova for pplss-tdTomato-KDEL. We thank Franz-Josef Müller (Kiel, Germany) for providing patient fibroblasts.

Author contributions $\mathrm{CK}$ and IK conceived the project. LB performed experiments and analyzed the data. CK designed experiments, analyzed data and wrote the paper with help of all authors. All authors discussed the results and implications at all stages.

\section{Compliance with ethical standards}

Conflict of interest The authors declare no conflict of interest.

OpenAccess This article is distributed under the terms of the Creative Commons Attribution 4.0 International License (http://creativeco mmons.org/licenses/by/4.0/), which permits unrestricted use, distribution, and reproduction in any medium, provided you give appropriate credit to the original author(s) and the source, provide a link to the Creative Commons license, and indicate if changes were made.

\section{References}

1. Hübner CA, Kurth I (2014) Membrane-shaping disorders: a common pathway in axon degeneration. Brain 137:3109-3121

2. Timmerman V, Clowes VE, Reid E (2013) Overlapping molecular pathological themes link Charcot-Marie-Tooth neuropathies and hereditary spastic paraplegias. Exp Neurol 246:14-25

3. Blackstone C (2012) Cellular pathways of hereditary spastic paraplegia. Annu Rev Neurosci 35:25-47

4. Rotthier A, Baets J, Timmerman V, Janssens K (2012) Mechanisms of disease in hereditary sensory and autonomic neuropathies. Nat Rev Neurol 8:73-85

5. Hu J, Shibata Y, Zhu PP, Voss C, Rismanchi N, Prinz WA, Rapoport TA, Blackstone C (2009) A class of dynamin-like GTPases involved in the generation of the tubular ER network. Cell 138:549-561

6. Orso G, Pendin D, Liu S, Tosetto J, Moss TJ, Faust JE, Micaroni M, Egorova A, Martinuzzi A, McNew JA et al (2009) Homotypic fusion of ER membranes requires the dynamin-like GTPase atlastin. Nature 460:978-983

7. Wang S, Tukachinsky H, Romano FB, Rapoport TA (2016) Cooperation of the ER-shaping proteins atlastin, lunapark, and reticulons to generate a tubular membrane network. eLife 5:e18605
8. Zhao X, Alvarado D, Rainier S, Lemons R, Hedera P, Weber CH, Tukel T, Apak M, Heiman-Patterson T, Ming L et al (2001) Mutations in a newly identified GTPase gene cause autosomal dominant hereditary spastic paraplegia. Nat Genet 29:326-331

9. Durr A, Camuzat A, Colin E, Tallaksen C, Hannequin D, Coutinho P, Fontaine B, Rossi A, Gil R, Rousselle C et al (2004) Atlastin1 mutations are frequent in young-onset autosomal dominant spastic paraplegia. Arch Neurol 61:1867-1872

10. Guelly C, Zhu PP, Leonardis L, Papic L, Zidar J, Schabhuttl M, Strohmaier H, Weis J, Strom TM, Baets J et al (2011) Targeted high-throughput sequencing identifies mutations in atlastin-1 as a cause of hereditary sensory neuropathy type I. Am J Hum Genet 88:99-105

11. Kornak U, Mademan I, Schinke M, Voigt M, Krawitz P, Hecht J, Barvencik F, Schinke T, Giesselmann S, Beil FT et al (2014) Sensory neuropathy with bone destruction due to a mutation in the membrane-shaping atlastin GTPase 3. Brain 137:683-692

12. Fischer D, Schabhuttl M, Wieland T, Windhager R, Strom TM, Auer-Grumbach M (2014) A novel missense mutation confirms ATL3 as a gene for hereditary sensory neuropathy type 1 . Brain 137:e286

13. Krols M, Detry S, Asselbergh B, Almeida-Souza L, Kremer A, Lippens S, De Rycke R, De Winter V, Muller FJ, Kurth I et al (2018) Sensory-neuropathy-causing mutations in ATL3 cause aberrant ER membrane tethering. Cell Rep 23:2026-2038

14. Kim KT, Moon Y, Jang Y, Lee KT, Lee C, Jun Y, Lee S (2017) Molecular mechanisms of atlastin-mediated ER membrane fusion revealed by a FRET-based single-vesicle fusion assay. Sci Rep 7:8700

15. Zhu PP, Patterson A, Lavoie B, Stadler J, Shoeb M, Patel R, Blackstone C (2003) Cellular localization, oligomerization, and membrane association of the hereditary spastic paraplegia $3 \mathrm{~A}$ (SPG3A) protein atlastin. J Biol Chem 278:49063-49071

16. Rismanchi N, Soderblom C, Stadler J, Zhu PP, Blackstone C (2008) Atlastin GTPases are required for Golgi apparatus and ER morphogenesis. Hum Mol Genet 17:1591-1604

17. Yan L, Sun S, Wang W, Shi J, Hu X, Wang S, Su D, Rao Z, Hu J, Lou Z (2015) Structures of the yeast dynamin-like GTPase Sey1p provide insight into homotypic ER fusion. J Cell Biol 210:961-972

18. Zhao G, Zhu PP, Renvoise B, Maldonado-Baez L, Park SH, Blackstone C (2016) Mammalian knock out cells reveal prominent roles for atlastin GTPases in ER network morphology. Exp Cell Res 349:32-44

19. Namekawa M, Muriel MP, Janer A, Latouche M, Dauphin A, Debeir T, Martin E, Duyckaerts C, Prigent A, Depienne C et al (2007) Mutations in the SPG3A gene encoding the GTPase atlastin interfere with vesicle trafficking in the ER/Golgi interface and Golgi morphogenesis. Mol Cell Neurosci 35:1-13

20. Pawar S, Ungricht R, Tiefenboeck P, Leroux JC, Kutay U (2017) Efficient protein targeting to the inner nuclear membrane requires Atlastin-dependent maintenance of ER topology. eLife 6:e28202

21. Zhao J, Hedera P (2013) Hereditary spastic paraplegia-causing mutations in atlastin-1 interfere with BMPRII trafficking. Mol Cell Neurosci 52:87-96

22. Klemm RW, Norton JP, Cole RA, Li CS, Park SH, Crane MM, Li L, Jin D, Boye-Doe A, Liu TY et al (2013) A conserved role for atlastin GTPases in regulating lipid droplet size. Cell Rep 3:1465-1475

23. Ulengin I, Park JJ, Lee TH (2015) ER network formation and membrane fusion by atlastin1/SPG3A disease variants. Mol Biol Cell 26:1616-1628

24. Jiang M, Chen $\mathrm{G}$ (2006) High $\mathrm{Ca}^{2+}$-phosphate transfection efficiency in low-density neuronal cultures. Nat Protoc 1:695-700 
25. Wacker I, Kaether C, Kromer A, Migala A, Almers W, Gerdes HH (1997) Microtubule-dependent transport of secretory vesicles visualized in real time with a GFP-tagged secretory protein. J Cell Sci 110:1453-1463

26. Nixon-Abell J, Obara CJ, Weigel AV, Li D, Legant WR, Xu CS, Pasolli HA, Harvey K, Hess HF, Betzig E et al (2016) Increased spatiotemporal resolution reveals highly dynamic dense tubular matrices in the peripheral ER. Science 354:aaf3928

27. Richter KN, Revelo NH, Seitz KJ, Helm MS, Sarkar D, Saleeb RS, D'Este E, Eberle J, Wagner E, Vogl C et al (2018) Glyoxal as an alternative fixative to formaldehyde in immunostaining and super-resolution microscopy. EMBO J 37:139-159

28. Lee WY, Goh G, Chia J, Boey A, Gunko NV, Bard F (2015) The ubiquitin ligase CBLC maintains the network organization of the Golgi apparatus. PLoS One 10:e138789

29. Fassler M, Li X, Kaether C (2011) Polar transmembrane-based amino acids in presenilin 1 are involved in endoplasmic reticulum localization, Pen2 protein binding, and gamma-secretase complex stabilization. J Biol Chem 286:38390-38396

30. Karanasios E, Stapleton E, Manifava M, Kaizuka T, Mizushima N, Walker SA, Ktistakis NT (2013) Dynamic association of the ULK1 complex with omegasomes during autophagy induction. J Cell Sci 126:5224-5238

31. Yonemura Y, Li X, Muller K, Kramer A, Atigbire P, Mentrup T, Feuerhake T, Kroll T, Shomron O, Nohl R et al (2016) Inhibition of cargo export at ER exit sites and the trans-Golgi network by the secretion inhibitor FLI-06. J Cell Sci 129:3868-3877

32. Ladner CL, Yang J, Turner RJ, Edwards RA (2004) Visible fluorescent detection of proteins in polyacrylamide gels without staining. Anal Biochem 326:13-20

33. Rivero-Gutierrez B, Anzola A, Martinez-Augustin O, de Medina FS (2014) Stain-free detection as loading control alternative to Ponceau and housekeeping protein immunodetection in western blotting. Anal Biochem 467:1-3

34. Gallione CJ, Rose JK (1985) A single amino acid substitution in a hydrophobic domain causes temperature-sensitive cell-surface transport of a mutant viral glycoprotein. J Virol 54:374-382

35. Toomre D, Keller P, White J, Olivo JC, Simons K (1999) Dualcolor visualization of trans-Golgi network to plasma membrane traffic along microtubules in living cells. J Cell Sci 112:21-33

36. Presley JF, Cole NB, Schroer TA, Hirschberg C, Zaal KJM, Lippincott-Schwartz J (1997) ER-to-Golgi transport visualized in living cells. Nature 389:81-85

37. Scales SJ, Pepperkok R, Kreis TE (1997) Visualization of ER-toGolgi transport in living cells reveals a sequential mode of action for COPII and COPI. Cell 90:1137-1148

38. Aridor M, Bannykh SI, Rowe T, Balch WE (1995) Sequential coupling between COPII and COPI vesicle coats in endoplasmic reticulum to Golgi transport. J Cell Biol 131:875-893

39. Berger J, Hauber J, Hauber R, Geiger R, Cullen BR (1988) Secreted placental alkaline phosphatase: a powerful new quantitative indicator of gene expression in eukaryotic cells. Gene 66:1-10

40. Misumi Y, Misumi Y, Miki K, Takatsuki A, Tamura G, Ikehara Y (1986) Novel blockade by brefeldin A of intracellular transport of secretory proteins in cultured rat hepatocytes. J Biol Chem 261:11398-11403

41. Zanetti G, Pahuja KB, Studer S, Shim S, Schekman R (2012) COPII and the regulation of protein sorting in mammals. Nat Cell Biol 14:20-28
42. Lorente-Rodriguez A, Barlowe C (2011) Entry and exit mechanisms at the cis-face of the Golgi complex. Cold Spring Harb Perspect Biol 3:a005207

43. Klumperman J, Schweizer A, Clausen H, Tang BL, Hong W, Oorschot V, Hauri HP (1998) The recycling pathway of protein ERGIC-53 and dynamics of the ER-Golgi intermediate compartment. J Cell Sci 111(Pt 22):3411-3425

44. Hauri HP, Kappeler F, Andersson H, Appenzeller C (2000) ERGIC-53 and traffic in the secretory pathway. J Cell Sci 113(Pt 4):587-596

45. Fan J, Hu Z, Zeng L, Lu W, Tang X, Zhang J, Li T (2008) Golgi apparatus and neurodegenerative diseases. Int $\mathrm{J}$ Dev Neurosci 26:523-534

46. Haase G, Rabouille C (2015) Golgi fragmentation in ALS motor neurons. New mechanisms targeting microtubules, tethers, and transport vesicles. Front Neurosci 9:448

47. Sanchez-Wandelmer J, Ktistakis NT, Reggiori F (2015) ERES: sites for autophagosome biogenesis and maturation? J Cell Sci 128:185-192

48. Kabeya Y, Mizushima N, Ueno T, Yamamoto A, Kirisako T, Noda T, Kominami E, Ohsumi Y, Yoshimori T (2000) LC3, a mammalian homologue of yeast Apg8p, is localized in autophagosome membranes after processing. EMBO J 19:5720-5728

49. Klionsky DJ, Abdalla FC, Abeliovich H, Abraham RT, AcevedoArozena A, Adeli K, Agholme L, Agnello M, Agostinis P, Aguirre-Ghiso JA et al (2012) Guidelines for the use and interpretation of assays for monitoring autophagy. Autophagy 8:445-544

50. Hu J, Rapoport TA (2016) Fusion of the endoplasmic reticulum by membrane-bound GTPases. Semin Cell Dev Biol 60:105-111

51. Okamoto M, Kurokawa K, Matsuura-Tokita K, Saito C, Hirata R, Nakano A (2012) High-curvature domains of the ER are important for the organization of ER exit sites in Saccharomyces cerevisiae. J Cell Sci 125:3412-3420

52. Zoppino FC, Militello RD, Slavin I, Alvarez C, Colombo MI (2010) Autophagosome formation depends on the small GTPase Rab1 and functional ER exit sites. Traffic 11:1246-1261

53. Graef M, Friedman JR, Graham C, Babu M, Nunnari J (2013) ER exit sites are physical and functional core autophagosome biogenesis components. Mol Biol Cell 24:2918-2931

54. Krols M, Asselbergh B, De Rycke R, De Winter V, Seyer A, Muller FJ, Kurth I, Bultynck G, Timmerman V, Janssens S (2018) Sensory neuropathy-causing mutations in ATL3 affect ER-mitochondria contact sites and impair axonal mitochondrial distribution. Hum Mol Genet. https://doi.org/10.1093/hmg/ddy352

55. Liang JR, Lingeman E, Ahmed S, Corn JE (2018) Atlastins remodel the endoplasmic reticulum for selective autophagy. J Cell Biol 217:3354-3367

56. Snapp EL, Sharma A, Lippincott-Schwartz J, Hegde RS (2006) Monitoring chaperone engagement of substrates in the endoplasmic reticulum of live cells. Proc Natl Acad Sci USA 103:6536-6541

Publisher's Note Springer Nature remains neutral with regard to jurisdictional claims in published maps and institutional affiliations. 